\title{
Recyclingpotenzial von gemischtem Gewerbeabfall
}

\author{
Martin Wellacher · Roland Pomberger
}

Online publiziert: 5. September 2017

(c) Der/die Autor(en) 2017. Dieser Artikel ist eine Open-Access-Publikation.

Zusammenfassung In Europa ist das Recycling von Abfällen in der Abfallrahmenrichtlinie vorgegeben und in Verordnungen und Richtlinien zu definierten Abfällen spezifiziert. Gemischter Gewerbeabfall ist Abfall, den ein gewerblicher Verursacher keiner getrennten Fraktion zugeführt hat. Über die Zusammensetzung von gemischtem Gewerbeabfall ist wenig bekannt. Gemischter Gewerbeabfall ist v. a. wegen seines hohen Kunststoffanteils und des niedrigen Wassergehalts für die Herstellung von Ersatzbrennstoffen (EBS) geeignet. Im Zuge der Aufbereitung in EBS-Anlagen werden Metalle abgetrennt, die wiederverwendet werden. Daraus ergibt sich eine Recyclingrate bei gemischten Gewerbeabfällen von 3 bis $5 \%$ bezogen auf die eingebrachten Abfälle. Das Ziel der vorliegenden Untersuchung war es, eine variable EBSAufbereitungs- und Recyclinganlage mit einer erhöhten Recyclingrate für gemischten Gewerbeabfall zu konzipieren, im Demonstrationsmaßstab zu erproben und einen Verfahrensvorschlag zu erarbeiten. Die Recyclingrate sollte von derzeit einstelligen Prozentzahlen auf zweistellige angehoben werden. Als Recyclingmaterialien aus gemischtem Gewerbeabfall wurden fünf Abfallarten bestimmt, für die es sowohl existierende Recyclingverfahren als auch einen Markt gibt, der eine Abnahme möglich und wirtschaftlich sinnvoll erscheinen lässt. Es wurden manuelle Sortieranalysen von gemischtem Gewerbeabfall hinsichtlich der fünf gewählten Recyclingmaterialien durchgeführt. Das für die Erprobung im Demonstrationsmaßstab gewählte Verfahren bestand aus der Abfolge Zerkleinerung, Magnetabscheidung, zweifacher Siebung, zweifacher ballistischer Separation und

\footnotetext{
Mag. Dr. M. Wellacher $(\varangle)$.

Univ.-Prof. DI Dr. R. Pomberger

Lehrstuhl für

Abfallverwertungstechnik und

Abfallwirtschaft, Montanuniversität Leoben, Franz Josef

Straße 18, 8700 Leoben, Österreich martin.wellacher@unileoben.ac.at
}

manueller Sortierung. Das Ergebnis aus den Sortieranalysen von gemischten Gewerbeabfällen ergab einen Anteil der fünf gewählten Recyclingmaterialien von $26 \%$. Die Funktionalität der Aufbereitungsaggregate bei der Erprobung im Demonstrationsmaßstab war gegeben. Hier konnte für gemischten Gewerbeabfall ein Recyclinganteil von 15\% abgetrennt werden. Die erzielte Recyclingrate für kommunalen Restabfall betrug $7 \%$. Ein Verfahrensvorschlag für eine variable EBS-Aufbereitungs- und Recyclinganlage mit erhöhter Recyclingrate wurde erarbeitet und die Qualität der abgetrennten Recyclingmaterialien wurde beurteilt.

\section{Recycling Potential of Mixed Industrial Waste}

Abstract Recycling of waste materials in Europe is governed by the EU Waste Directive 2008 and for certain waste materials specified in further directives and regulations. Mixed industrial waste is waste which was not separated into recyclable fractions by the industrial producer. There is few knowledge available about the composition of mixed industrial waste. This waste is suitable for the production of refuse derived fuel (RDF) mainly because of its high plastic and low water content. In the course of its processing in RDF plants metals are separated to be recycled. Thus a recycling rate of $3-5 \%$ related to the input is realized. The aim of this research was firstly to design a variable RDF treatment and recycling plant with an elevated recycling rate for mixed industrial waste, secondly to test the concept on a demonstration scale and thirdly to develop a process proposal. The recycling rate should be raised above $10 \%$. Five recycling materials were defined out of mixed industrial waste which on the one hand can be processed with an existing recycling technology and on the other hand can be marketed economically. Manual sorting analysis for the five materials was performed. The chosen process for the demonstration tests consisted in shredding, magnetic separation, double screening, double ballistic separation and manual sorting. The sorting analysis resulted a share of $27 \%$ of the five recycling materials in mixed industrial waste. The tested machines in the demonstration scale worked well. A recycling rate of $15 \%$ for mixed industrial waste could be realized. However for residual municipal solid waste it resulted in $7.0 \%$. A process proposal for a variable RDF treatment and recycling plant with elevated recycling rate was presented and the qualities of the separated recycling materials were assessed.

\section{Einleitung}

Das Recycling von Abfällen ist ein Gebot unserer Zeit. Ein industriell seit langem angewendetes Recyclingverfahren für Eisenschrotte ist das LinzDonawitz-Verfahren der Stahlindustrie, bei welchem Roheisen mit Anteilen von Eisenschrott zu neuwertigem Stahl verarbeitet wird. Solche Verfahren gilt es auf Abfälle anzuwenden, die derzeit noch deponiert oder verbrannt werden (Bundesgesetz 2008).

Die Rahmenbedingungen für Recycling sind unterschiedlich. Einerseits müssen eine Technologie und eine $\mathrm{Ab}$ satzmöglichkeit dafür verfügbar sein, andererseits muss es für den Abfallbesitzer einen wirtschaftlichen Anreiz oder eine gesetzliche Verpflichtung dazu geben. In Europa ist das Recycling von Abfällen in der Abfallrahmenrichtlinie (Europäische Union 2008) vorgegeben und in Verordnungen und Richtlinien zu definierten Abfällen spezifiziert, z. B. der Elektroaltgeräterichtlinie (Europäische Union 2012).

\subsection{Recycling}

Recycling bedeutet Wiederverwertung eines chemischen Stoffes, d. h. von Elementen, Molekülen und Gemischen. Das kann entweder für den ursprünglichen Zweck oder für andere Zwecke passieren.

In Österreich befindet sich die Abfallwirtschaft in einer Übergangsphase 


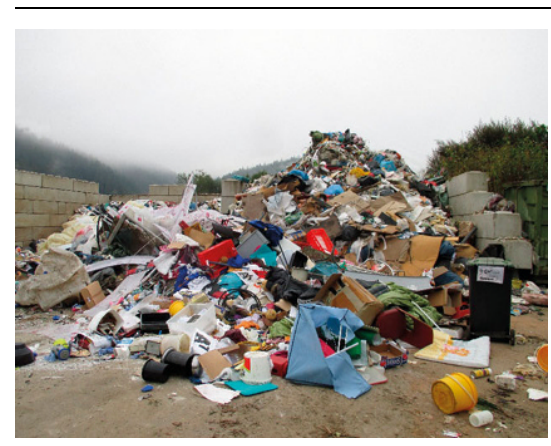

Abb. 1 Gemischter Gewerbeabfall

von einer thermischen Verwertungswirtschaft in Richtung einer stofflichen (Pomberger et al. 2017). Während sich das Recycling bei Kunststoffen, Altholz oder Aushubmaterialien zögerlich entwickelt, ist es bei Glas, Papier, biogenen Abfällen oder Eisenmetallen fortgeschrittener. Bei manchen Abfällen gibt es auch Rückschritte vom Recycling, z. B. bei Klärschlamm.

Dass Recycling in unterschiedlicher Qualität stattfinden kann, wird gesetzlich noch nicht berücksichtigt. Beispielsweise wird Eisenschrott sowohl zur Herstellung von hochwertigem Fahrzeugstahl als auch von minderwertigem Baustahl stofflich verwertet. Die beiden Verwertungsverfahren werden gleich gewichtet, nur die Höhe der Recyclingraten wird in Betracht gezogen.

Das Recycling unterschiedlicher Abfälle bewirkt auch Unterschiede in deren Umweltauswirkungen. Beispielsweise spart das Recycling einer Tonne Aluminiumschrott ein Vielfaches an Energie und Treibhausgasemissionen gegenüber dem Recycling einer Tonne Altglas (Frischenschlager et al. 2010).

Die Berechnung von Recyclingraten in der EU ist auf verschiedene Arten zulässig (Europäische Union 2011). Weil diese nicht gut vergleichbar sind, gibt es eine Diskussion über eine Vereinheitlichung (Europäische Union 2015).

Recyclingraten gibt es in Europa beispielsweise für Verpackungsabfälle (Europäische Union 1994), Elektroaltgeräte (Europäische Union 2012) oder Altbatterien (Europäische Union 2006). Auch für die gesamten Siedlungsabfälle werden Recyclingraten diskutiert (Europäische Union 2015). Über das Recycling von gemischtem Gewerbeabfall gibt es keine derartige Diskussion.

Das Recycling von Abfällen ist auch abhängig von der Vorsortierung und der Sammlung. So steigt die Qualität

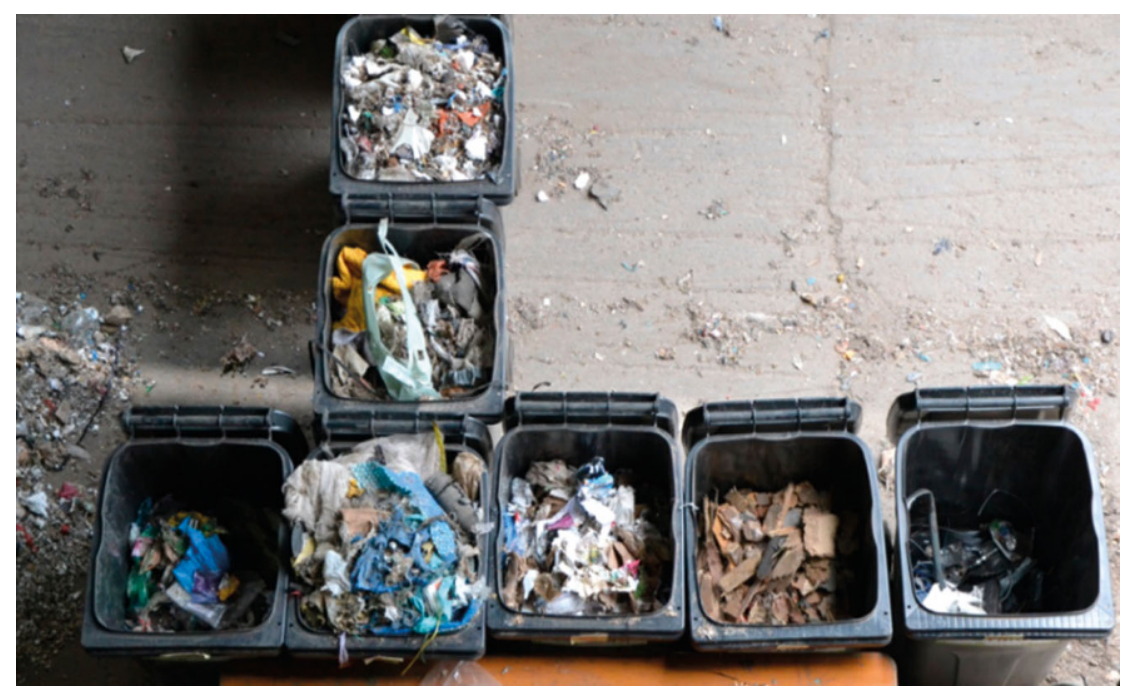

Abb. 2 Sortierte Anteile aus einer von drei Sortieranalysen, von rechts nach links: Altmetalle, Altholz, PPK, Restfraktion (PET und Inert nicht abgebildet)

von Recyclingprodukten mit der Differenzierung und Qualität der Quellensortierung.

\subsection{Gemischter Gewerbeabfall}

Gemischter Gewerbeabfall ist nicht-getrennt erfasster und nicht-kommunaler Restabfall, es ist Abfall, den der gewerbliche Verursacher keiner getrennten Fraktion zugeführt hat. Das können beispielsweise gemischte Baustellenabfälle, Büroabfälle oder Industrieabfälle sein. Sie werden auch als Hausmüllähnlicher Gewerbeabfall bezeichnet (Abb. 1).

Über die Zusammensetzung von gemischtem Gewerbeabfall ist wenig bekannt. Auch über das Aufkommen von gemischtem Gewerbeabfall in Österreich und anderen EU-Mitgliedstaaten herrscht Unklarheit. Im Entwurf zum aktuellen Bundesabfallwirtschaftsplan wird dazu von Siedlungsabfällen aus Haushalten und ähnlichen Einrichtungen gesprochen, wobei unklar bleibt, ob auch gemischter Gewerbeabfall damit gemeint ist oder nicht (BMLFUW 2017). Für einzelne Regionen wurde dieser Abfall mit 30 bis $70 \%$ des Aufkommens von gemischtem Siedlungsabfall geschätzt (Amt der Steiermärkischen Landesregierung 2010; Pomberger und Gungl 2010). Nicht zuletzt auch aus diesen Gründen gibt es wenige Untersuchungen zum Recycling von gemischtem Gewerbeabfall.

In Österreich wird gemischter Gewerbeabfall in den letzten Jahren überwiegend zu Ersatzbrennstoff (EBS) auf- bereitet (Pomberger 2007; Pomberger und Sarc 2014).

\subsection{Verfahren für die Herstellung von Ersatzbrennstoffen}

Gemischter Gewerbeabfall ist v. a. wegen seines hohen Kunststoffanteils und des niedrigen Wassergehalts für die Herstellung von EBS geeignet. In diesen Anlagen werden auch kommunaler Restabfall, Sortierreste, Überkorn aus Splittinganlagen oder andere heizwertreiche Materialien zu EBS aufbereitet.

Im Zuge der mechanischen Aufbereitung werden Störstoffe abgetrennt, darunter Metalle, die anschließend stofflich verwertet werden. Neben der Herstellung von EBS ist das ein Synergieeffekt, der seit langem zu einer mehr oder weniger wirksamen Metallabscheidung in diese Anlagen führt. Dabei handelt es sich zu 90 bis $95 \%$ um Magnetschrott und zu 5 bis $10 \%$ aus nicht-magnetischem Schrott. Die abgetrennten Metalle führen so zu einer Recyclingrate bei gemischten Gewerbeabfällen von 3 bis $5 \%$. Auch andere Störstoffe werden abgeschieden, aber nicht stofflich verwertet. Dazu zählen Steine, Keramik, schwere Kunststoffteile oder chlorhaltige Kunststoffe.

Die angewendeten Verfahren sind meist mechanisch und magnetisch. $\mathrm{Zu}$ den mechanischen Verfahren zählen Zerkleinerung, Siebung, Windsichtung und ballistische Separation. Zu den magnetischen Verfahren zählen Magnete und Wirbelstromabscheider. Darüber hinaus werden sensorgestützte Sortier- 


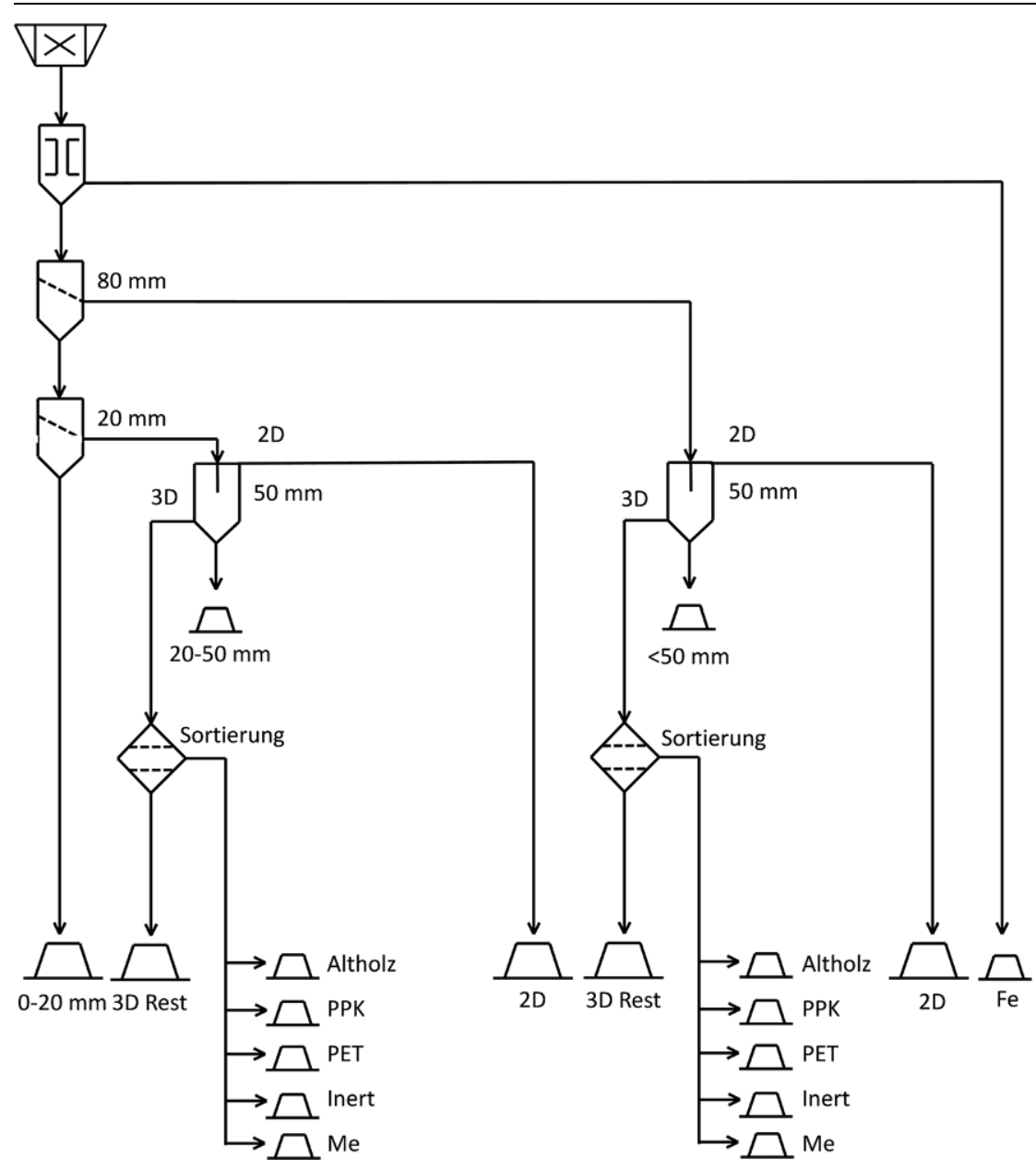

Abb. 3 Erprobtes Verfahren im Demonstrationsmaßstab zur Erhöhung der Recyclingrate von gemischtem Gewerbeabfall (Me Metalle, Fe magnetische Metalle)

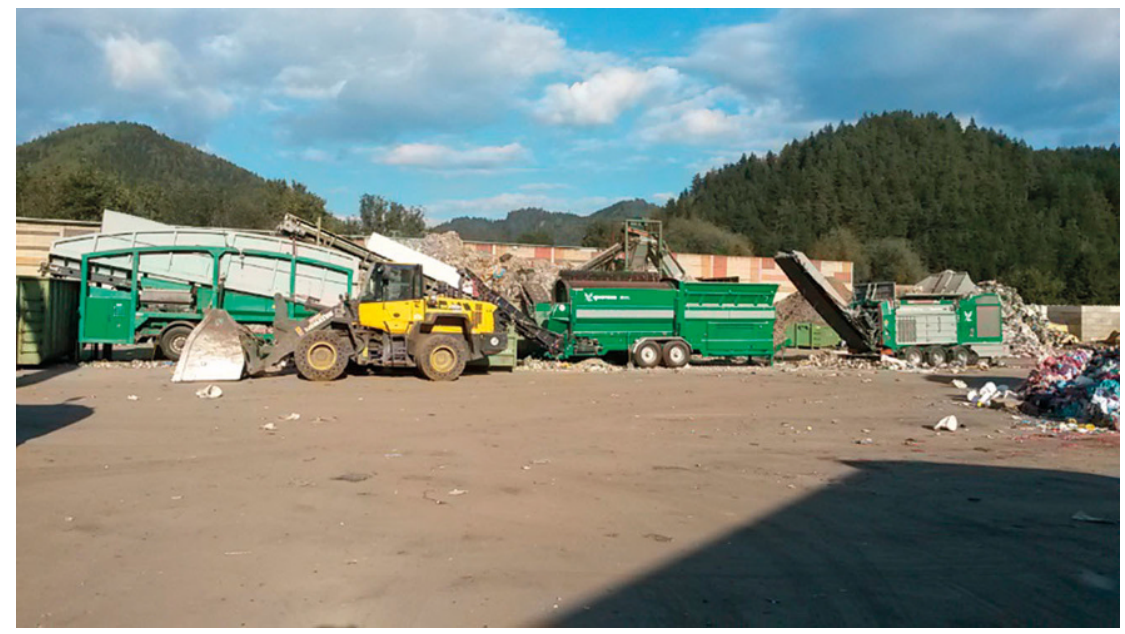

Abb. 4 Aufbereitungsaggregate für den Demonstrationsversuch; von rechts nach links: Zerkleinerer mit Magnetabscheider, Trommelsieb und ballistischer Separator maschinen auf der Basis der Nah-Infrarot-Messung (NIR) als Chlorschleusen eingesetzt.

\subsection{Ziel der Untersuchung}

Ziel der Untersuchung war es, eine variable EBS-Aufbereitungs- und Recyclinganlage mit einer erhöhten Recyclingrate für gemischten Gewerbeabfall zu konzipieren, im Demonstrationsmaßstab $\mathrm{zu}$ erproben und einen Verfahrensvorschlag zu erarbeiten. Die Recyclingrate sollte von derzeit einstelligen Prozentzahlen auf zweistellige angehoben werden.

\section{Material und Methoden}

Als Recyclingmaterialien aus gemischtem Gewerbeabfall wurden fünf Abfallarten bestimmt, für die es sowohl existierende Recyclingverfahren als auch einen Markt gibt, der eine Abnahme möglich und wirtschaftlich sinnvoll erscheinen lässt:

- Altholz für die Spanplattenherstellung,

- Altpapier, Altpappe und Altkarton für die Papier- und Kartonherstellung (PPK),

- PET-Flaschen für das PET-Recycling (PET),

- Altmetalle für verschiedene Metallrecyclingprozesse, v. a. in der Eisenund Aluminiumindustrie und

- Inertmaterialien Keramik, Glas, Stein, Beton für die Ablagerung auf Baurestmassendeponien bzw. das Baustoffrecycling (Inert).

\subsection{Zusammensetzung von gemischtem Gewerbeabfall}

Es wurden manuelle Sortieranalysen von gemischtem Gewerbeabfall hinsichtlich der fünf gewählten Recyclingmaterialien durchgeführt. Vom Material wurden an drei Tagen im Abstand von mehreren Wochen Sortierkampagnen mit etwa $1 \mathrm{~m}^{3}$ Material durchgeführt, welches über den Zeitraum von $4 \mathrm{~h}$ aus dem laufenden vorzerkleinerten Strom einer EBS-Aufbereitungsanlage entnommen wurde (Abb. 2).

\subsection{Demonstrationsversuch}

Das gewählte Verfahren zur Erprobung bestand aus der Abfolge Zerkleinerung, Magnetabscheidung, zweifache Siebung, zweifache ballistische Separation und manuelle Sortierung (Abb. 3). 


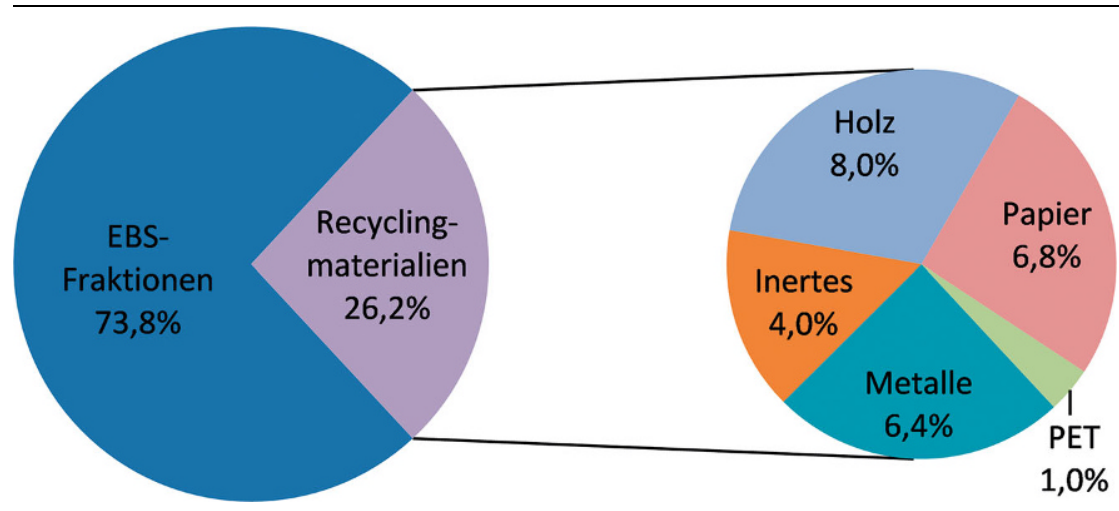

Abb. 5 Potenzial von fünf Recyclingmaterialien in gemischten Gewerbeabfällen (Mittelwerte aus drei Sortierkampagnen)

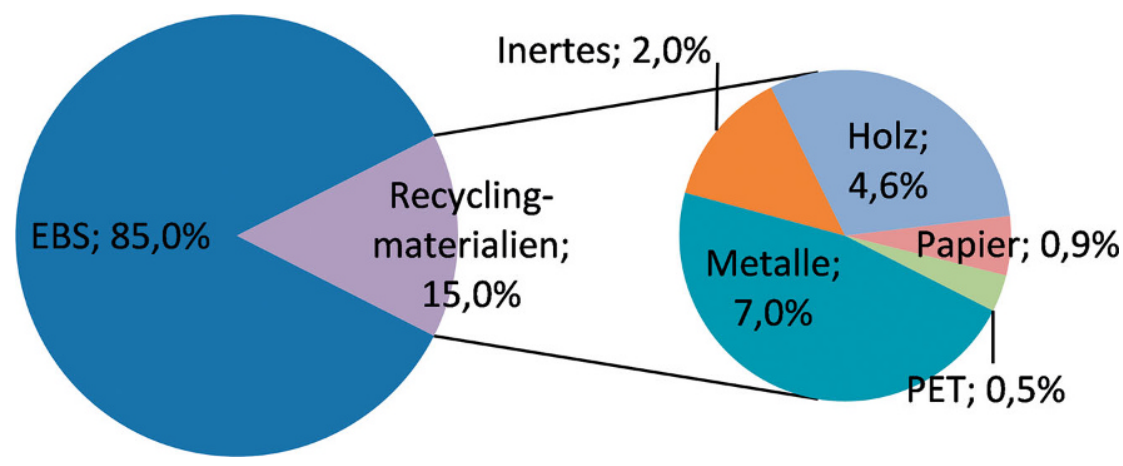

Abb. 6 Anteil der beim Demonstrationsversuch gewonnenen fünf Recyclingmaterialien aus gemischtem Gewerbeabfall

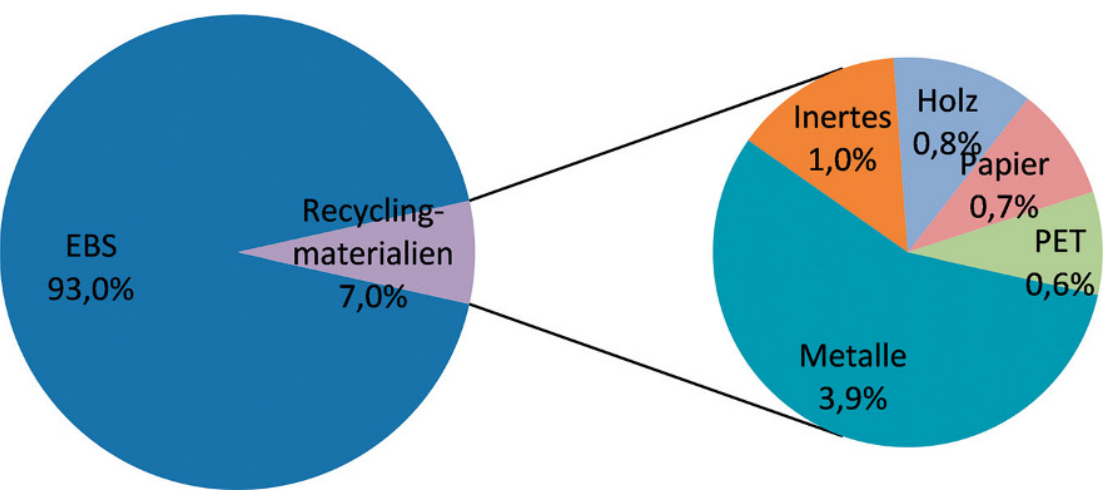

Abb. 7 Anteil der beim Demonstrationsversuch gewonnenen fünf Recyclingmaterialien aus kommunalem Restabfall

Die erprobten Inputmaterialien waren gemischter Gewerbeabfall und kommunaler Restabfall. Sie wurden getrennt behandelt.

Die Festlegung der Trenngröße bei der Siebung und der ballistischen Separation wurde mit $80 \mathrm{~mm}$ für die erste Siebstufe, $20 \mathrm{~mm}$ für die zweite sowie $50 \mathrm{~mm}$ für die erste und zweite ballistische Separation gewählt.

Die Versuche im Demonstrationsmaßstab fanden mit drei Aggregaten
Siebung, ballistischer Separation und händischer Sortierung durchgeführt.

Jeder Durchlauf wurde über $3 \mathrm{~h}$ betrieben.

Alle eingesetzten Maschinen waren Produkte der Komptech GmbH, Frohnleiten. Es handelte sich um Mobilmaschinen mit Dieselantrieb und Fahrgestell.

Als Vorzerkleinerer wurde ein „Terminator 5000 SF ec Trailer" verwendet. Die Walze hatte eine Länge von $3000 \mathrm{~mm}$, einen Durchmesser von $1050 \mathrm{~mm}$ und wurde mit einer Drehzahl von $32 \mathrm{~min}^{-1}$ betrieben.

Als Magnetabscheider war ein Überbandmagnet am Austragsband des Vorzerkleinerers im Querstrom angebracht.

Als Trommelsieb wurde eine "MAXX“ mit zwei Siebtrommeln mit 80-mm-Rundloch bzw. 20-mm-Quadratloch verwendet. Die Siebtrommeln hatten eine Fläche von $22,5 \mathrm{~m}^{2}$, eine Länge von $4500 \mathrm{~mm}$ und einen Durchmesser von $1800 \mathrm{~mm}$.

Als ballistischer Separator wurde ein „Ballistor mobil“ verwendet. Die vier gegengleich rotierenden Paddel hatten eine Neigung von $10^{\circ}$ und eine Rundlochung von $50 \mathrm{~mm}$. Es wurden drei Fraktionen getrennt: flache zweidimensionale Fraktion (2D), kubische dreidimensionale Fraktion (3D) und Siebunterkorn $<50 \mathrm{~mm}$. Die Feineinstellung erfolgte durch die Rotationsgeschwindigkeit und den Neigungswinkel der Paddel so, dass die 3D-Fraktion visuell keine 2D-Materialien (Folien) mehr enthielt und umgekehrt.

Die erzeugten Fraktionen wurden über die gesamte Durchlaufzeit in Containern aufgefangen und ihr Gewicht auf einer Brückenwaage gewogen.

Die Sortierung der 3D-Fraktionen fand manuell in einer Sortierkabine mit Sortierband und Sortierplätzen statt. Das Gewicht aller sortierten Fraktionen wurde auf einer Brückenwaage bestimmt. Von den 3D-Fraktionen wurden jeweils mehrere $\mathrm{m}^{3}$ beprobt und sortiert, bis von jeder Recyclingfraktion etwa $0,2 \mathrm{~m}^{3}$ vorhanden waren.

In einem Nebenversuch wurden die beiden 3D-Fraktionen aus dem Demonstrationsversuch mit einem Magnet-Trommelabscheider im Technikum der IFE Aufbereitungstechnik $\mathrm{GmbH}$ in Waidhofen/Ybbs magnetabgeschieden.

Die sortierten Recyclingmaterialien wurden einer visuellen Beurteilung auf ihre Eignung für einen Recyclingprozess beurteilt. Altholz wurde von einem 


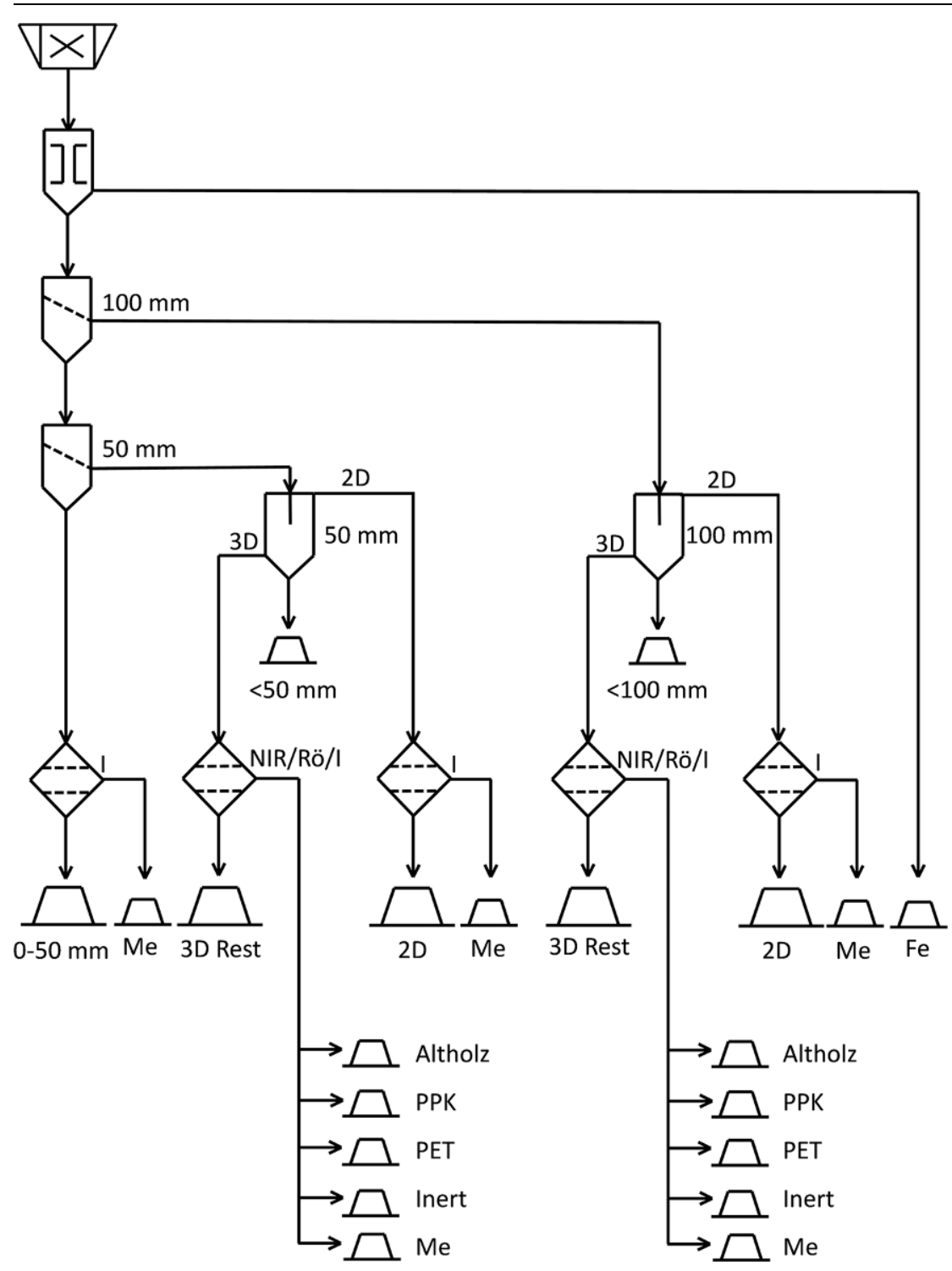

Abb. 8 Verfahrensvorschlag EBS-Anlage miterhöhter Recyclingrate(NIR Nahinfrarot, Rö Röntgen, / Induktion, Me Metalle, Fe magnetische Metalle)

Hersteller von Spanplatten begutachtet, Metalle von einem Recyclingbetrieb für Schrotte sowie PET von einem PETRecycler.

\section{Ergebnisse}

\subsection{Zusammensetzung von gemischtem Gewerbeabfall}

Die Sortieranalysen von gemischtem Gewerbeabfall ergaben einen Anteil der fünf gewählten Recyclingmaterialien von $26 \%$. Davon entfielen $8 \%$ auf Altholz, $7 \%$ auf PPK, $6 \%$ auf Altmetalle, $4 \%$ auf Inertes und $1 \%$ auf PET (Abb. 5). Diese Anteile stellten das Potenzial für jedoch waren nach wenigen Betriebsstunden Siebtrommel und Reinigungsbürste intensiv verschmutzt und verlegt. Die zweite Siebstufe bei $20 \mathrm{~mm}$ arbeitete einwandfrei und zeigte keine Verschmutzungsneigung.

Bei der ersten Stufe der ballistischen Separation, die mit Material $>80 \mathrm{~mm}$ beschickt wurde, war nach einem Betriebstag eine moderate Reinigung etwa eines Drittels der Siebbleche nötig. Diese hatten sich v. a. mit Textilien verhängt. In der zweiten Stufe, die mit Material $<80 \mathrm{~mm}$ beschickt wurde, blieben die Siebbleche unbeeinträchtigt. Die ballistisch separierten 3D-Fraktionen waren einfach vereinzelbar, d.h. es gab kein Zusammenhängen durch Drähte, Kabel oder Schnüre. Damit waren diese Fraktionen sowohl gut sortierbar als auch gut magnetisch bearbeitbar.

Im Nebenversuch zum Metallausbringen mit einem Magnet-Trommelabscheider aus der 3D-Fraktion wurden das Ausbringen mit $95 \%$ und die Reinheit mit $91 \%$ bestimmt.

\subsubsection{Recyclingrate für gemischtem Gewerbeabfall}

Im Versuch konnte für gemischten Gewerbeabfall ein Recyclinganteil von $15 \%$ abgetrennt werden (Abb. 6). Dieser wurden zu $41 \%$ über den Magnetabscheider nach der Zerkleinerung und zu $59 \%$ aus den 3D-Fraktionen gewonnen.

Damit konnten $57 \%$ des theoretischen Recyclingpotenzials realisiert werden, bezogen auf die im Input sortierten 26,2\% Recyclingmaterialien (Abb. 5). Mit dem Versuch konnte nachgewiesen werden, dass in diesen 3DFraktionen ein Konzentrieren der fünf Recyclingmaterialien von $21 \%$ (nach Abzug der magnetischen Fraktion) auf $27(>80 \mathrm{~mm})$ bzw. $33 \%$ (50 bis $80 \mathrm{~mm}$ ) stattfand.

Die durch Magnetabscheidung und Sortierung gewonnen Metalle waren mehr $(7,0 \%)$, als an Potenzial in den Ausgangsmaterialien festgestellt wurde $(6,4 \%)$. Das war darauf zurückzuführen, dass die mittels Überbandmagnet abgeschiedene Fraktion auch einen Anteil von nicht-metallischen Teilen ausgetragen hatte, der das Ergebnis verfälschte, weil er zu den Metallen mitgezählt wurde. Außerdem konnten Metalle visuell noch in anderen Outputmaterialien (2D-Fraktion, 0 - bis 20mm-Fraktion) erkannt werden, die mit diesem Versuchsaufbau nicht separiert werden konnten. 


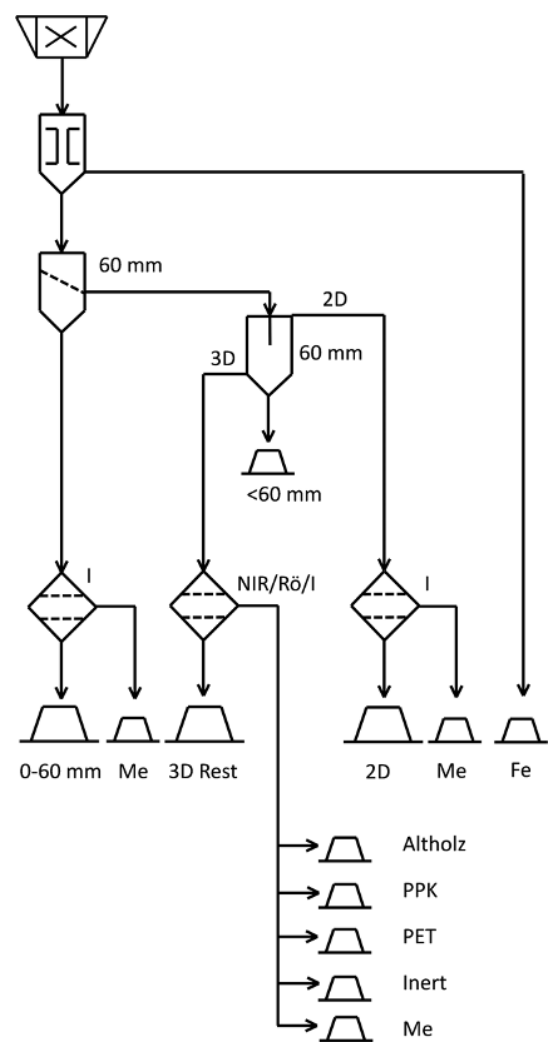

Abb. 9 Verfahrensvorschlag EBSAnlage mit erhöhter Recyclingrate (Minimalvariante) (NIR Nahinfrarot, Rö Röntgen, I Induktion, Me Metalle, Fe magnetische Metalle)

\subsubsection{Recyclingrate für kommunalen Restabfall}

Die erzielte Recyclingrate für kommunalen Restabfall betrug 7,0\% (Abb. 7). Die wurden zu $44 \%$ mit dem Überbandmagnet beim Zerkleinerer und $\mathrm{zu}$ $56 \%$ aus den 3D-Fraktionen separiert.

\subsection{Verfahrensvorschlag EBS-Anlage mit erhöhter Recyclingrate}

Das vorgeschlagene Verfahren wird hier in zwei Varianten beschrieben. Gibt es keine Einschränkungen, besteht das Verfahren aus den Schritten Zerkleinerung, Siebung bei $100 \mathrm{~mm}$, ballistische Separation bei $>100 \mathrm{~mm}$, Siebung bei $50 \mathrm{~mm}$, ballistische Separation bei 50 bis $100 \mathrm{~mm}$ und sensorgestützten Sortiermaschinen auf Basis von Induktion, NIR und Röntgenstrahlung (Abb. 8).

Eine Minimalvariante des vorgeschlagenen Verfahrens könnte aus Zerkleinerung, Siebung bei $60 \mathrm{~mm}$, ballistischer Separation bei $>60 \mathrm{~mm}$ und manueller Sortierung bestehen (Abb. 9).

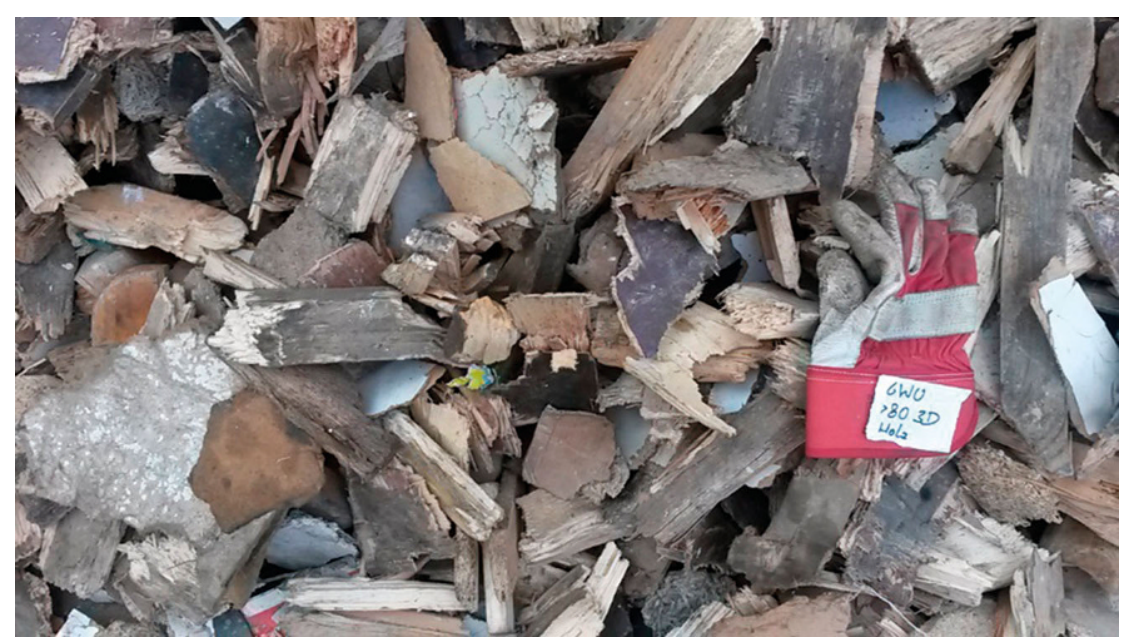

Abb. 10 Sortiertes Altholz aus gemischtem Gewerbeabfall (3D-Fraktion)

Die gewählten Trenngrößen der Trommelsiebe beim Demonstrationsversuch waren nicht ideal, da das $\mathrm{Zu}$ sammenspiel mit den 50-mm-Lochgrößen der ballistischen Separatoren nicht gegeben war. So wurde das $<80-\mathrm{mm}$ Material zuerst bei $20 \mathrm{~mm}$ gesiebt und das Überkorn 20 bis $80 \mathrm{~mm}$ im ballistischen Separator wieder bei $50 \mathrm{~mm}$ gesiebt. Im ballistischen Separator entstand so eine große Menge Unterkorn, das schon mit der Siebung hätte abgeschieden werden können, wenn dieses statt mit einer 20-mm-Lochung mit einer 50-mm-Lochung ausgestattet gewesen wäre. Die Conclusio daraus lautet, dass bei der vorgeschlagenen Abfolge die Trenngrößen in Sieben und ballistischen Separatoren übereinstimmen sollten.

Der sich daraus ergebende naheliegende Verzicht auf eine Siebung zwischen Vorzerkleinerung und ballistischer Separation wurde an einzelnen Anlagen bereits realisiert (Zawadzki 2012). Dem erhöhten Reinigungsaufwand des ballistischen Separators ist dann jedoch Rechnung zu tragen. Eine Aufbereitung von feuchten Abfällen, z. B. kommunalem Restabfall, ist mit dieser Variante nicht zu empfehlen, da diese die Lochplatten des ballistischen Separators innerhalb kurzer Zeit zusetzen können.

Die ballistische Separation ist für jedes Material und Aufbereitungsziel über den Neigungswinkel, die Rotationsgeschwindigkeit der Paddel und den Aufgabepunkt zu justieren. Das sollte auf einer Recyclinganlage einfach durchführbar sein.
Beim Demonstrationsversuch wurde festgestellt, dass trotz Magnetabscheidung nach der Zerkleinerung alle nachfolgend abgetrennten Fraktionen nach wie vor magnetische Metalle im Prozentbereich enthielten, deren Gewinnung möglich gewesen wäre. Daher wird hier eine zusätzliche Output-nahe Metallabscheidung gewählt.

In diesem Verfahrensvorschlag sind sensorgestützte Sortiermaschinen vorgeschlagen, die für die Metallseparation mit Induktionsmessung arbeiten, für PET, Holz, PPK mit NIR-Reflexionsmessung und für Inertmaterialien mit Röntgentransmissionsmessung.

Häufig diskutiert wird eine Siebung vor der Vorzerkleinerung. Auf manchen EBS-Anlagen wurde eine solche Vorsiebung realisiert (Welsch 2016). Sie soll der Entlastung der Vorzerkleinerung dienen, indem diese kein Material bekommt, das nicht zerkleinert werden muss, weil es schon unter der Trenngröße des nachfolgenden Siebaggregates liegt. So könnte der Vorzerkleinerer mit kleinerer Kapazität ausgelegt werden und zusätzlich einem geringeren Verschleiß unterliegen. Diesen Vorteilen ist der Aufwand eines zusätzlichen Siebaggregats mit Fördertechnik, Förderhöhenverlust und Bauraum gegenüberzustellen.

Auf die für die weitergehende EBSHerstellung nötigen Aggregate Feinzerkleinerer, sensorgestützte NIR-Sortiermaschine als Chlorschleuse und Feinsiebe wurde hier nicht eingegangen. 


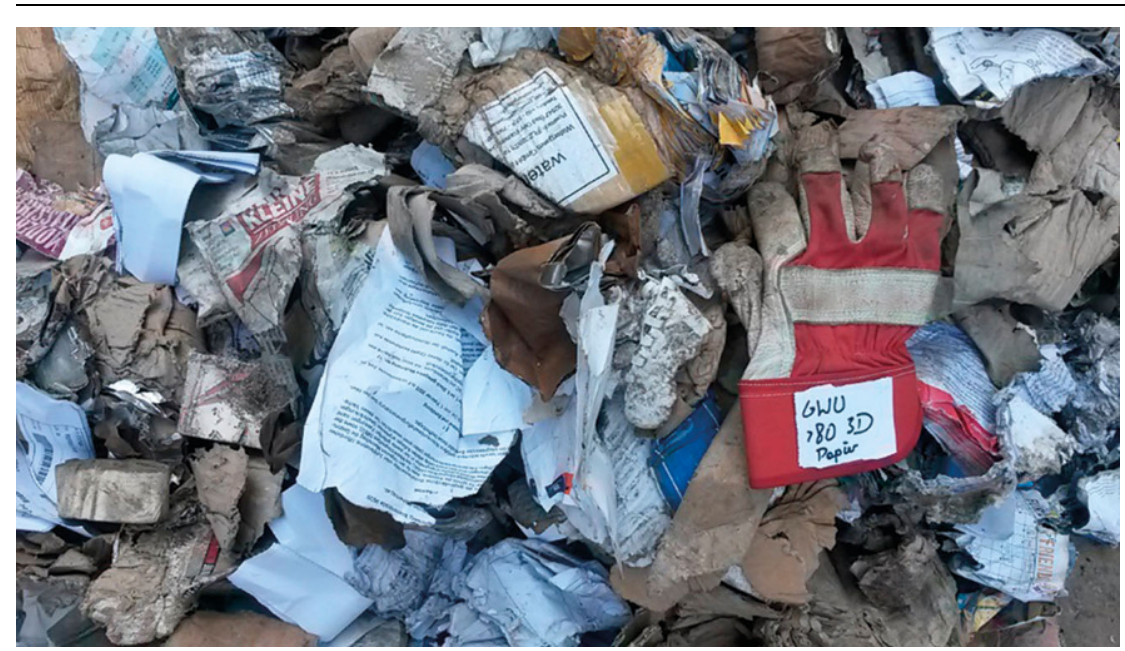

Abb. 11 Sortiertes Altpapier aus gemischtem Gewerbeabfall (3D-Fraktion)

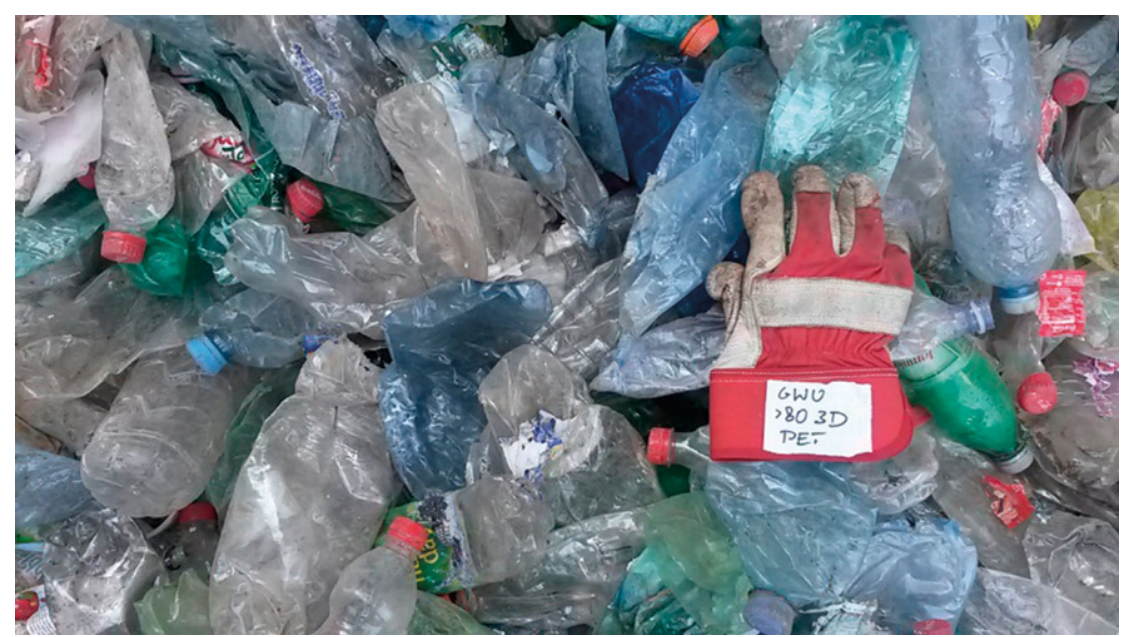

Abb. 12 Sortierte PET-Flaschen aus gemischtem Gewerbeabfall (3D-Fraktion)

\subsection{Qualität der Recyclingmaterialien}

Das sortierte Altholz (Abb. 10) wurde von einem Hersteller von Spanplatten als nicht interessant bezeichnet. Begründet wurde das mit der dunklen Färbung und dem modrigen Geruch.

Das sortierte Altpapier erschien visuell für ein Recycling geeignet (Abb. 11).

Das sortierte PET wurde von einem Recyclingbetrieb für PET-Flaschen mit einer Angabe des erzielbaren Erlöses als geeignet bezeichnet (Abb. 12).

Die sortierten Inertmaterialien sind visuell zur Ablagerung auf einer Baurestmassen-Deponie geeignet (Abb. 13).

Die Metalle wurden von einem Recyclingbetrieb für gemischte Metallabfälle als gut geeignet befundet (Abb. 14).

\section{Diskussion}

Das Ziel der Untersuchung, eine variable EBS-Aufbereitungs- und Recyclinganlage mit einer erhöhten Recyclingrate für gemischten Gewerbeabfall zu konzipieren, im Demonstrationsmaßstab zu erproben und einen Verfahrensvorschlag für eine Anlage zu erarbeiten, womit die Recyclingrate von derzeit einstelligen Prozentzahlen auf zweistellige erhöht werden sollte, konnte erreicht werden. Die Recyclingrate konnte auf $15 \%$ erhöht werden. Das wurde im Demonstrationsmaßstab nachgewiesen.

\subsection{Vorgeschlagene Verfahren}

Soll die Metallabscheidung optimiert werden, ist eine solche bei allen erzeugten Fraktionen durchzuführen.
Sensorgestützte Sortiermaschinen, die über Induktionsspulen Metalle erkennen, sind eine gute Möglichkeit diese abzutrennen. Inzwischen sind diese ähnlich wirtschaftlich wie konventionelle Kombinationen von Magneten und Wirbelstromabscheidern, sofern bestimmte Korngrößenbereiche eingehalten werden.

Den Trenngrößen bei den Siebschritten kommt eine besondere Bedeutung zu. Einerseits wirken sie zusammen mit den Lochgrößen der ballistischen Separatoren und den Arbeitsbereichen nachfolgender Sortiermaschinen. Andererseits kann damit der Aufwand der Feinzerkleinerung reduziert werden, wenn die vorgegebenen Korngrößen für EBS-Material ohne weitere Zerkleinerung erreicht werden können, z. B. $<100 \mathrm{~mm}$ für Wirbelschichtmaterial oder $<60 \mathrm{~mm}$ für Kalzinator-Material. Um verschiedene Abfallarten aufbereiten zu können und flexibel die Produktausbeute und -qualität zu verändern, wäre eine rasche Verstellbarkeit der Lochgrößen interessant. Solche Maschinen sind derzeit nur als Sternsiebe verfügbar, die aber aus anderen Gründen nicht für gemischten Gewerbeabfall geeignet sind. Die Entwicklung solcher Maschinen wird hier angeregt.

Die ballistische Separation ist bekannt als Schlüsseltechnologie für die Aufbereitung von Recyclingfraktionen aus gemischten Abfällen. Die Konzentration der gewählten Recyclingfraktionen aus gemischtem Gewerbeabfall und kommunalem Restabfall in den 3D-Fraktionen konnte in dem vorliegenden Versuch nachgewiesen und quantifiziert werden.

Windsichter werden derzeit oft in EBS-Anlagen eingesetzt. Da sie keine Konzentrierung von Recyclingmaterialien herbeiführen, haben sie bei einer Intensivierung des Recyclings Nachteile gegenüber ballistischen Separatoren. Trotzdem kann die Anwendung von Windsichtern von Bedeutung sein. Die Störstoffabtrennung aus der 3D-RestFraktion nach der Sortierung und vor der Feinzerkleinerung zum EBS kann derzeit am besten durch Windsichter durchgeführt werden.

Sensorgestützte Sortiermaschinen für die 3D-Fraktion können hohe Recyclingraten und hohe Qualitäten von Recyclingmaterialien realisieren. Gleichzeitig erhöhen sie den Invest und die Komplexität jeder Anlage durch das notwendige Umfeld (Materialverteilung, Beschleunigungsband, Druck- 


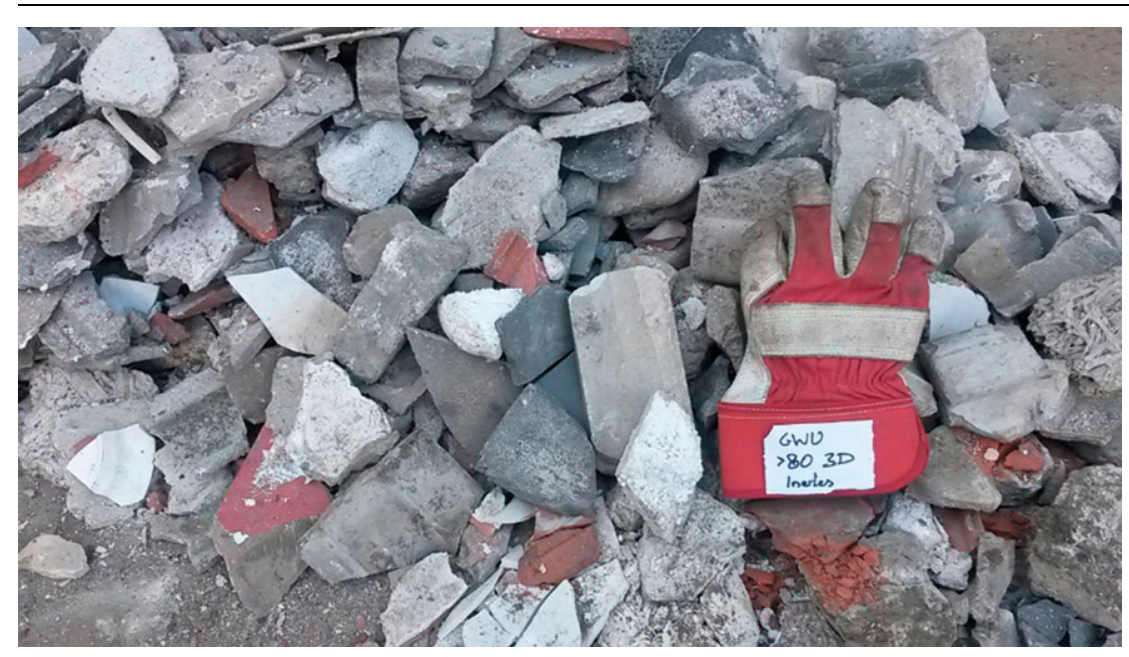

Abb. 13 Sortierte Inertmaterialien aus gemischtem Gewerbeabfall (3D-Fraktion)

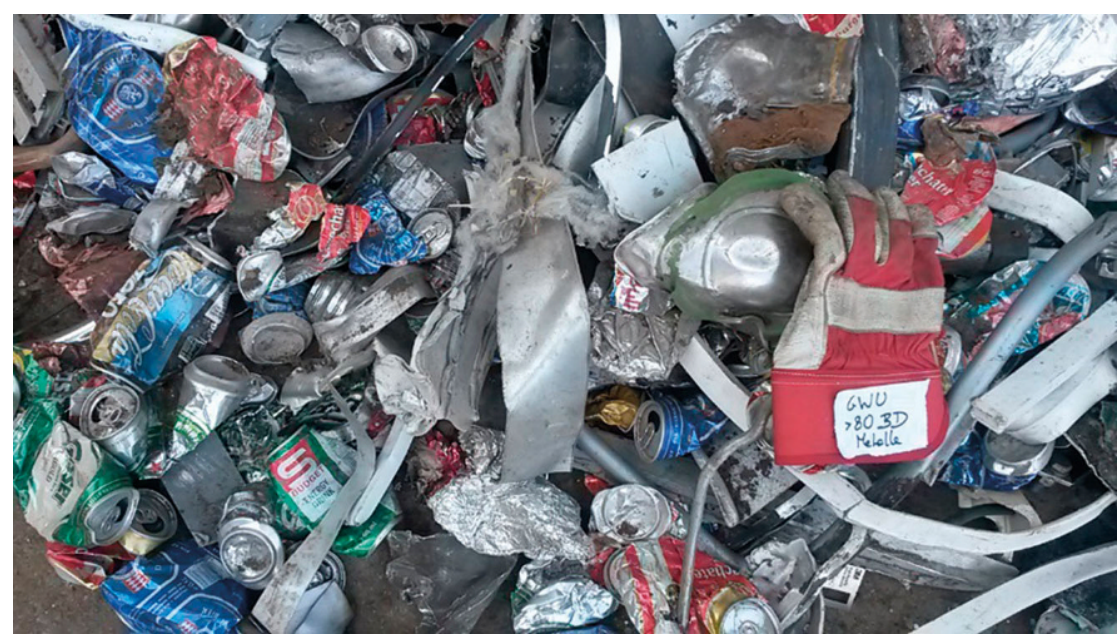

Abb. 14 Sortierte Metalle aus gemischtem Gewerbeabfall (3D-Fraktion)

luftversorgung, Förderhöhenverluste, Bauraum etc.).

\subsection{Recyclingmaterialien}

Die erzielbare Recyclingrate von gemischtem Gewerbeabfall ist deutlich höher als jene von kommunalem Restabfall.

Die hier gewählten fünf Recyclingmaterialien haben einen realistischen Zugang zum Recyclingmarkt. Darüber hinaus gibt es andere potenzielle Recyclingmaterialien, z. B. Polyethylenverpackungen oder Glas. Jedoch ist es nicht bei jeder Abfallart sinnvoll, ein Recycling anzustreben, z. B. chlorhaltigen oder gealterten Kunststoffen.

Sowohl die sortierte PET-Fraktion als auch die sortierten Altmetalle wurden in ihrer Verwertbarkeit geprüft und als geeignet befunden. noch von der Herstellung von Baustoffen stammen, sollte einer Ablagerung eine rechtliche Klärung vorausgehen.

\subsection{Umsetzung der Ergebnisse in der österreichischen Abfallwirtschaft}

Die Umsetzung der Erfahrungen aus der vorliegenden Untersuchung in Form einer Neukonzeption einer EBSRecyclinganlage oder eines Umbaus einer EBS-Anlage in nächster Zukunft ist nur vereinzelt $\mathrm{zu}$ erwarten. Grund dafür sind die fehlenden Vorgaben der Abfallgesetzgebung für einen Vorzug des Recyclings vor der Verbrennung und die fehlenden wirtschaftlichen Anreize der Märkte v.a. für Altholz und Inertmaterialien. Für Altmetalle, PET und PPK sollten die Marktumstände bereits interessant genug sein. Außerhalb von Österreich gibt es solche Anlagen, wie z.B. in Albstadt, Deutschland (Korn 2016), oder in Zilina, Slowakei (Wellacher 2013).

Eine zumindest teilweise Umsetzung wäre technisch schon vor zehn Jahren möglich gewesen (Hribernik 2007; Wellacher und Pretzler 2008).

\subsection{Grenzen der vorliegenden Arbeit}

Gemischter Gewerbeabfall zeichnet sich durch eine besonders heterogene Zusammensetzung aus, die naturgemäß von den Branchen der entsorgten Betriebe bestimmt wird. Die Untersuchung umfasst zwar ein breites Spektrum an gewerblichen Produzenten, aber nur eine begrenzte Region der österreichischen Entsorgungslandschaft. Daher könnte eine Übertragung auf andere Regionen zu abweichenden Ergebnissen führen.

Das Marktinteresse der sortierten Recyclingmaterialien aus den aufbereiteten gemischten Gewerbeabfällen wurde nicht in jener Breite erhoben, die einer Investitionsentscheidung vorausgehen sollte. Hier müssten tiefergehende Untersuchungen stattfinden.

Danksagung Die Autoren bedanken sich bei der Komptech GmbH und einem anderen Unternehmen, das hier nicht genannt werden möchte, für die Finanzierung der dargestellten Untersuchungen.

Funding Open access funding provided by Montanuniversität Leoben. 
Open Access Dieser Artikel wird unter der Creative Commons Namensnennung 4.0 International Lizenz (http:// creativecommons.org/licenses/by/4.

0/deed.de) veröffentlicht, welche die
Nutzung, Vervielfältigung, Bearbeitung, Verbreitung und Wiedergabe in jeglichem Medium und Format erlaubt, sofern Sie den/die ursprünglichen $\mathrm{Au}$ tor(en) und die Quelle ordnungsgemäß nennen, einen Link zur Creative Commons Lizenz beifügen und angeben, ob Änderungen vorgenommen wurden.

\section{Literatur}

Amt der Steiermärkischen Landesregierung (2010): Landes-Abfallwirtschaftsplan Steiermark 2010. Fachabteilung 19D, Bürgergasse 5a, $810 \mathrm{Graz}$

Bundesgesetz (2008): Verordnung des Bundesministers für Land- und Forstwirtschaft, Umwelt und Wasserwirtschaft über Deponien (Deponieverordnung 2008 - DVO 2008). Bundesgesetzblatt für die Republik Österreich II/39/2008 Bundesministerium für Land- und Forstwirtschaft, Umwelt und Wasserwirtschaft (BMLFUW) (2017): Entwurf Bundes-Abfallwirtschaftsplan 2017 Teil 1. BMLFUW Stubenring 1, 1010 Wien

Europäische Union (1994): Richtlinie 94/62/EG des Europäischen Parlaments und des Rates vom 20. Dezember 1994 über Verpackungen und Verpackungsabfälle

Europäische Union (2006): Richtlinie 2006/66/ EG des Europäischen Parlaments und des Rates vom 6. September 2006 über Batterien und Akkumulatoren sowie Altbatterien und Altakkumulatoren und zur Aufhebung der Richtlinie 91/157/EWG

Europäische Union (2008): Richtlinie 2008/98/ EG des Europäischen Parlamentes und des Rates vom 19. November 2008 über Abfälle Europäische Union (2011): Beschluss der Kommission vom 18. November 2011 mit Vorschriften und Berechnungsmethoden für die Über- prüfung der Einhaltung der Zielvorgaben gemäß Artikel 11 Absatz 2 der Richtlinie 2008/98/EG des Europäischen Parlaments und des Rates (Bekannt gegeben unter Aktenzeichen K(2011) 8165) $(211 / 753 / \mathrm{EU})$

Europäische Union (2012): Richtlinie 2012/19EU des Europäischen Parlamentes und des Rates vom 4. Juli 2012 über Elektro- und ElektronikAltgeräte

Europäische Union (2015): Closing the loop - An EU action plan for the Circular Economy (EU Circular Economy Package 2015) (COM(2015)614)

Frischenschlager, H., Karigl, B., Lampert, C., Pölz, W., Schindler, I., Tesar, M., Wiesenberger, H. \& Winter, B. (2010): Klimarelevanz ausgewählter Recycling-Prozesse in Österreich - Endbericht. Umweltbundesamt GmbH, Spittelauer Lände 5, 1090 Wien

Hribernik, P. (2007): Gewerbeabfall Recycling Stand der Technik und Entwicklungspotentiale. Diplomarbeit am FH Jonanneum, Kapfenberg Korn, A. (2016): Erklärungen im Zuge der Besichtigung der Abfallbehandlungsanlage Albstadt, Deutschland. Korn Recycling GmbH, Albstadt, Deutschland

Pomberger, R. (2007): Ersatzbrennstoffe aus Siedlungsabfällen - Anforderungen, Möglichkeiten, Betriebserfahrungen. ÖWAV Abfallwirtschaft als Ressourcenlieferant der Zukunft?
Pomberger, R., Gungl, E. (2010): Neue Wege der Bewirtschaftung von Gewerbeabfallgemischen in der Steiermark (Österreich). ReSource 4:5 Pomberger, R., Sarc, R. (2014): Solid Alternative Fuels - legal, technological and economical developments in Austria. ZKG International 4: 56-64

Pomberger, R., Sarc, R. \& Lorber K.E. (2017): Dynamic visualisation of municipal waste management performance in the EU using Ternary Diagram method. Waste Management 61: 558-571

Wellacher, M. (2013): Zilina RDF Production Plant, Slovakia. 5. Internationale Tagung MBA, Sortierung und Recycling: 66-72

Wellacher, M. \& Pretzler, R. (2008): A cold process. Waste Management World 2008: 22-25 Welsch, D. (2016): Erklärungen im Zuge der Besichtigung der Abfallbehandlungsanlage Resitejo, Portugal. Masias Recycling S.L., Sant Joan les Fonts, Spanien

Zawadzki, J. (2012): Erklärungen im Zuge der Besichtigung der Abfallbehandlungsanlage Myslenice, Polen. AGREX-ECO SP. Z.O.O., Warsaw, Polen 